\title{
NUMERICAL METHODS FOR SINGULARLY PERTURBED ELLIPTIC PROBLEMS CONTAINING TWO PERTURBATION PARAMETERS ${ }^{1}$
}

E. O'RIORDAN ${ }^{1}$, M. L. PICKETT ${ }^{2}$, and G. I. SHISHKIN ${ }^{3}$

\section{${ }_{1,2}$ Dublin City University}

School of Mathematical Sciences, Dublin City University, Ireland

E-mail: eugene.oriordan@dcu.ie; maria.pickett2@mail.dcu.ie

${ }^{3}$ Institute of Mathematics and Mechanics

Russian Academy of Sciences, Ekaterinburg, Russia

E-mail: shishkin@imm.uran.ru

Received October 15, 2005; revised April 04, 2006; published online May 25, 2006

\begin{abstract}
A priori parameter explicit bounds on the derivatives of the solution of a two parameter singularly perturbed elliptic problem in two space dimensions are presented. These bounds are used to establish parameter uniform error bounds for a numerical method consisting of upwinding on a tensor product of two piecewise uniform meshes.
\end{abstract}

Key words: Elliptic, two parameters, a priori bounds

\section{Introduction}

When analysing the convergence behaviour of numerical approximations to the solution of a singularly perturbed differential equation involving two singular perturbation parameters (denoted here by $\varepsilon$ and $\mu$ ), it is worth noting that the error is a function of three parameters: the mesh parameter $N$ (the number of mesh elements used in each coordinate direction) and the two perturbation parameters. Parameter-uniform numerical methods [2] are methods such that the pointwise error $E(N ; \varepsilon, \mu)$ is bounded independently of both perturbation parameters. Parameter-uniform methods for two-parameter

\footnotetext{
${ }^{1}$ This research was supported in part by the National Center for Plasma Science and Technology Ireland and by the Russian Foundation for Basic Research grant No. 04-01-00578.
} 
problems based on fitted operator methods on uniform meshes are given in $[10,11]$. More recently, fitted piecewise-uniform meshes have been used to generate parameter-uniform methods for two-parameter ordinary differential equations $[7,8]$ and for singularly perturbed parabolic equations [6]. In this paper, we examine a two-parameter elliptic problem in two space dimensions.

Consider the following class of singularly perturbed elliptic problems posed on the unit square $\Omega=(0,1)^{2}$,

$$
\begin{aligned}
& L_{\varepsilon, \mu} u=\varepsilon\left(u_{x x}+u_{y y}\right)+\mu\left(a_{1} u_{x}+a_{2} u_{y}\right)-b u=f \quad \text { in } \Omega, \\
& \left.u\right|_{\Gamma_{B}}=s_{1}(x),\left.\quad u\right|_{\Gamma_{T}}=s_{2}(x),\left.\quad u\right|_{\Gamma_{L}}=q_{1}(y),\left.\quad u\right|_{\Gamma_{R}}=q_{2}(y), \\
& s_{1}(0)=q_{1}(0), s_{2}(0)=q_{1}(1), s_{1}(1)=q_{2}(0), s_{2}(1)=q_{2}(1), \\
& a_{1}(x, y) \geq \alpha_{1}>0, a_{2}(x, y) \geq \alpha_{2}>0, b(x, y) \geq 2 \beta>0,
\end{aligned}
$$

where $\Gamma_{B}, \Gamma_{T}, \Gamma_{L}$ and $\Gamma_{R}$ are the edges of the boundary $\partial \Omega$ defined by

$$
\begin{array}{ll}
\Gamma_{B}=\{(x, 0) \mid 0 \leq x \leq 1\}, & \Gamma_{T}=\{(x, 1) \mid 0 \leq x \leq 1\}, \\
\Gamma_{L}=\{(0, y) \mid 0 \leq y \leq 1\}, & \Gamma_{R}=\{(1, y) \mid 0 \leq y \leq 1\} .
\end{array}
$$

Throughout this paper, we assume sufficient regularity and compatibility on the data so that the solution and its components are sufficiently smooth for the following analysis to be valid. With respect to regularity assume that

$$
a_{1}, a_{2}, b, f \in C^{n, \lambda}(D), \lambda \in(0,1), s_{1}, s_{2}, q_{1}, q_{2} \in C^{m}(J),
$$

where $D, J$ are open sets such that $\bar{\Omega} \subset D,[0,1] \subset J$ and $n, m$ are sufficiently large for our analysis. In this paper, the norm $\|v\|_{R}=\max _{\vec{x} \in R}|v(\vec{x})|$ is the maximum pointwise norm. Throughout this paper $C$ is a generic constant which is independent of the singular perturbation parameters $\varepsilon, \mu$ and the mesh parameters $N, M$.

Note that the differential equation (1.1a) contains two singular perturbation parameters $0<\varepsilon \leq \varepsilon_{0}=\mathcal{O}(1)$ and $0 \leq \mu \leq 1$. We let

$$
\alpha=\min \left\{\alpha_{1}, \alpha_{2}\right\}, \quad \gamma<\min \left\{\frac{b}{2 a_{1}}, \frac{b}{2 a_{2}}\right\} .
$$

The analysis for this two-parameter problem naturally splits into two cases, $\mu^{2} \leq \frac{\gamma \varepsilon}{\alpha}$ and $\mu^{2} \geq \frac{\gamma \varepsilon}{\alpha}$. In the case of $\mu^{2} \leq \frac{\gamma \varepsilon}{\alpha}$, the analysis is similar to the reaction-diffusion problem [1] when $\mu=0$ and boundary layers of width $\mathcal{O}(\sqrt{\varepsilon})$ appear in the neighbourhood of all four edges. For the case of $\mu^{2} \geq \frac{\gamma \varepsilon}{\alpha}$ the analysis is more intricate and boundary layers of width $\mathcal{O}\left(\frac{\varepsilon}{\mu}\right)$ appear in the neighbourhood of the edges $x=0, y=0$ and boundary layers of width $\mathcal{O}(\mu)$ appear in the neighbourhood of $x=1, y=1$.

In this paper, we confine the discussion to the case of

$$
\mu^{2} \leq \frac{\gamma \varepsilon}{\alpha}
$$




\section{Bounds on the Solution $u$ and its Derivatives}

In this section we establish a priori bounds on the derivatives of the solution of (1.1). These bounds are essential for the error analysis in subsequent sections. We begin by stating a continuous minimum principle for the differential operator in (1.1). The proof of this comparison principle is standard.

Lemma 1. If $w \in C^{2}(\Omega) \cap C^{0}(\bar{\Omega})$ such that $\left.L_{\varepsilon, \mu} w\right|_{\Omega} \leq 0$ and $\left.w\right|_{\partial \Omega} \geq 0$, then $\left.w\right|_{\bar{\Omega}} \geq 0$.

An immediate consequence of this Lemma is the stability bound:

$$
\|u\| \leq\|s\|_{\Gamma_{B} \cup \Gamma_{T}}+\|q\|_{\Gamma_{L} \cup \Gamma_{R}}+\frac{1}{2 \beta}\|f\| .
$$

The next lemma establishes parameter-explicit bounds on the derivatives of the solution. Within the realm of singularly perturbed problems, the proof is essentially classical except that here it is applied in the case of a two parameter problem.

Lemma 2. If $f \in C^{1, \lambda}(\bar{\Omega}), s, q \in C^{3, \lambda}(0,1)$ are independent of $\varepsilon$ and $\mu$, and assuming sufficient compatibility of the boundary data at the corners, the derivatives of the solution of (1.1) satisfy the following bounds for all nonnegative integers $k$ and $m$, where $1 \leq k+m \leq 3$

$$
\left\|\frac{\partial^{k+m} u}{\partial x^{k} \partial y^{m}}\right\| \leq C\left(\frac{1}{\sqrt{\varepsilon}}\right)^{k+m}(1+\|u\|),
$$

where $C$ is independent of the parameters $\varepsilon$ and $\mu$.

Proof. Note that we can write $\omega=u-g$, where $\omega$ satisfies an equation similar to (1.1) with homogeneous boundary conditions. We have

$$
L_{\varepsilon, \mu} \omega=f-L_{\varepsilon, \mu} g=\hat{f} \quad \text { on } \Omega, \quad \omega \equiv 0 \quad \text { on } \partial \Omega .
$$

Consider the transformation

$$
\xi=\frac{(\mu+\sqrt{\varepsilon}) x}{\varepsilon}, \quad \eta=\frac{(\mu+\sqrt{\varepsilon}) y}{\varepsilon} .
$$

The transformed domain $\tilde{\Omega}$ is given by $\tilde{\Omega}=\left(0, \frac{\mu+\sqrt{\varepsilon}}{\varepsilon}\right)^{2}$. Applying this transformation, the above differential equation becomes

$$
\tilde{\omega}_{\xi \xi}+\tilde{\omega}_{\eta \eta}+\frac{\mu}{\sqrt{\varepsilon}+\mu} \tilde{a_{1}} \tilde{\omega}_{\xi}+\frac{\mu}{\sqrt{\varepsilon}+\mu} \tilde{a_{2}} \tilde{\omega}_{\eta}-\frac{\varepsilon}{(\sqrt{\varepsilon}+\mu)^{2}} \tilde{b} \tilde{\omega}=\tilde{f}, \quad \text { on } \tilde{\Omega},
$$

where $\tilde{\omega}(\xi, \eta)=\omega(x, y)$. The coefficients $\tilde{a_{1}}, \tilde{a_{2}}, \tilde{b}$ are defined similarly and

$$
\tilde{f}(\xi, \eta)=\frac{\varepsilon}{(\sqrt{\varepsilon}+\mu)^{2}} \hat{f}(x, y) .
$$


For each $\left(\zeta_{1}, \zeta_{2}\right) \in \tilde{\Omega}$, we denote the rectangle $(\zeta-\delta, \zeta+\delta)^{2} \cap \tilde{\Omega}$ by $\tilde{R}_{\delta}\left(\zeta_{1}, \zeta_{2}\right)$, where $\delta=\mathcal{O}(1)$. For all $(\xi, \eta) \in \tilde{\Omega}$ and $\tilde{R}_{\delta}$ we have (see [4, page 110] or [5, Theorem 3.1]) that

$$
|\tilde{\omega}|_{1, \lambda, \tilde{R}_{\delta}} \leq C\left(\|\tilde{f}\|_{0, \lambda, \tilde{R}_{2 \delta}}+\|\tilde{\omega}\|_{\tilde{R}_{2 \delta}}\right)
$$

and for $l=0,1$

$$
|\tilde{\omega}|_{l+2, \lambda, \tilde{R}_{2 \delta}} \leq C\left(\|\tilde{f}\|_{l, \lambda, \tilde{R}_{2 \delta}}+\|\tilde{\omega}\|_{\tilde{R}_{2 \delta}}\right)
$$

where $|\cdot|_{l+2, \lambda, \tilde{R}_{\delta}}$ and $\|\cdot\|_{l+1, \lambda, \tilde{R}_{\delta}}$ are the standard semi-norms and norms in $C^{k, \lambda}$ (see, for example, $[4,5]$ ). Since $|\omega|_{k, \Omega} \leq|\omega|_{k, \lambda, \Omega}$, we obtain

$$
|\tilde{\omega}|_{1, \tilde{R}_{\delta}} \leq|\omega|_{1, \lambda, \tilde{R}_{\delta}} \leq C\left(\|\tilde{f}\|_{0, \lambda, \tilde{R}_{2 \delta}}+\|\tilde{\omega}\|_{\tilde{R}_{2 \delta}}\right),
$$

and for $l=0,1$

$$
|\tilde{\omega}|_{l+2, \tilde{R}_{\delta}} \leq|\omega|_{l+2, \lambda, \tilde{R}_{\delta}} \leq C\left(\|\tilde{f}\|_{l, \lambda, \tilde{R}_{2 \delta}}+\|\tilde{\omega}\|_{\tilde{R}_{2 \delta}}\right) .
$$

Transforming back to the original variables this implies for all $(x, y) \in \Omega$ and $R_{\delta}=R_{\delta}(x, y)$

$$
\left(\frac{\varepsilon}{\mu+\sqrt{\varepsilon}}\right)|\omega|_{1, R_{2 \delta}} \leq C\left(\frac{\varepsilon}{(\mu+\sqrt{\varepsilon})^{2}}\left(\frac{\varepsilon}{\mu+\sqrt{\varepsilon}}\right)^{\lambda}\|\hat{f}\|_{0, \lambda, R_{2 \delta}}+\|\omega\|_{R_{2 \delta}}\right),
$$

and for $l=0,1$

$$
\begin{aligned}
& \left(\frac{\varepsilon}{\mu+\sqrt{\varepsilon}}\right)^{l+2}|\omega|_{l+2, R_{\delta}} \leq C \frac{\varepsilon}{(\mu+\sqrt{\varepsilon})^{2}} \\
& \quad \times\left(\sum_{v=0}^{l}\left(\frac{\varepsilon}{\mu+\sqrt{\varepsilon}}\right)^{v}|\hat{f}|_{v, R_{2 \delta}}+\left(\frac{\varepsilon}{\mu+\sqrt{\varepsilon}}\right)^{l+\lambda}|\hat{f}|_{l, \lambda, R_{2 \delta}}\right)+C\|\omega\|_{R_{2 \delta}} .
\end{aligned}
$$

Replacing $\hat{f}$ by $f-L_{\varepsilon, \mu} g$ yields the required result.

Remark 1. The proof in Lemma 2 is applicable in the case where the positivity constraint $(1.1 \mathrm{~d})$ is relaxed to

$$
a_{1}(x, y) \geq 0, \quad a_{2}(x, y) \geq 0, \quad b(x, y) \geq 2 \beta>0 .
$$

\section{Regular Component}

In order to obtain more informative parameter explicit error bounds on the derivatives of the solution of (1.1), the solution is decomposed into a sum of regular and layer components. The extension of idea from [9] is used to define the regular solution, which avoids imposing overly artificial compatibility conditions. We show that there exists a function $v$ such that $L_{\varepsilon, \mu} v=f$ and when its boundary conditions are chosen appropriately, the function $v$ and its derivatives up to second order are bounded independently of the small parameters. 
Define the zero order differential operator $L_{0}$ to be

$$
L_{0} z=-b z \text {. }
$$

Consider the extended domain $\Omega^{*}=(-d, 1+d) \times(-d, 1+d) \supset \bar{\Omega}, d>0$. The extended differential operators $L_{\varepsilon, \mu}^{*}$ and $L_{0}^{*}$ coincide with the operators $L_{\varepsilon, \mu}$ and $L_{0}$ respectively on $\Omega$. Below, we implicitly define smooth extensions $a_{1}^{*}, a_{2}^{*}, b^{*}$ and $f^{*}$ of the functions $a_{1}, a_{2}, b$ and $f$ to $\Omega^{*}$ so that they coincide with the functions $a_{1}, a_{2}, b$ and $f$ in $\bar{\Omega}$. These extensions are constructed so that $a_{1}^{*} \geq 0, a_{2}^{*} \geq 0, b^{*} \geq 2 \beta>0$ at all points in $\Omega^{*}$ and

$$
f^{*}=a_{1}^{*}=a_{2}^{*}=0, \quad b^{*}=2 \beta, \quad(x, y) \in \Omega^{*} \backslash D,
$$

where $D$ is an open set such that $\bar{\Omega} \subset D \subset \Omega^{*}$.

Consider the differential equation $L_{\varepsilon, \mu}^{*} v^{*}=f^{*}$ on $\Omega^{*}$ and decompose $v^{*}$ as follows

$$
v^{*}(x, y)=v_{0}^{*}(x, y)+\sqrt{\varepsilon} v_{1}^{*}(x, y)+\varepsilon v_{2}^{*}(x, y),
$$

where

$$
\begin{aligned}
& L_{0}^{*} v_{0}^{*}=f^{*}, \quad \sqrt{\varepsilon} L_{0}^{*} v_{1}^{*}=\left(L_{0}^{*}-L_{\varepsilon, \mu}^{*}\right) v_{0}^{*}, \\
& \varepsilon L_{\varepsilon, \mu}^{*} v_{2}^{*}=\sqrt{\varepsilon}\left(L_{0}^{*}-L_{\varepsilon, \mu}^{*}\right) v_{1}^{*},\left.\quad v_{2}^{*}\right|_{\partial \Omega^{*}}=0 .
\end{aligned}
$$

Note that $v_{0}^{*}$ and $v_{1}^{*}$ satisfy zero order differential equations and hence there are no issues of compatibility. The term $v_{2}^{*}$ is the solution of an elliptic problem on the extended domain $\Omega^{*}$. The extensions $b^{*}, f^{*}$ are such that the function $g^{*} \equiv\left(L_{0}^{*}-L_{\varepsilon, \mu}^{*}\right) v_{1}^{*}$ is zero at the four corners of the extended domain and $g^{*} \in C^{1, \lambda}\left(\bar{\Omega}^{*}\right)$. In this way the term $v_{2} \in C^{3, \lambda}\left(\bar{\Omega}^{*}\right)$ is sufficiently regular for our purposes [3].

Given $\mu^{2} \leq \frac{\gamma \varepsilon}{\alpha}$, we see that the functions $v_{0}^{*}$ and $v_{1}^{*}$ and their derivatives are bounded independently of both small parameters. Since $v_{2}^{*}$ satisfies a similar equation to (1.1) we can use Lemma 1 and Lemma 2 along with $\mu^{2} \leq \frac{\gamma \varepsilon}{\alpha}$ to obtain for $0 \leq k+m \leq 3$

$$
\left\|\frac{\partial^{k+m} v_{2}^{*}}{\partial x^{k} \partial y^{m}}\right\| \leq C\left(\frac{1}{\sqrt{\varepsilon}}\right)^{k+m}, \quad \text { if } \quad \mu^{2} \leq \frac{\gamma \varepsilon}{\alpha} .
$$

Define the regular component $v$ to be the solution of the elliptic problem

$$
L_{\varepsilon, \mu} v=f,(x, y) \in \Omega, \quad \text { and } \quad v=v^{*},(x, y) \in \partial \Omega .
$$

Assuming sufficient smoothness of the coefficients, we can establish the following bounds on the first three derivatives of the regular component $v$

$$
\left\|\frac{\partial^{k+m} v}{\partial x^{k} \partial y^{m}}\right\| \leq C\left(1+\varepsilon^{(2-k-m) / 2}\right), \quad 0 \leq k+m \leq 3, \quad \text { if } \mu^{2} \leq \frac{\gamma \varepsilon}{\alpha} .
$$




\section{Layer Components}

Associated with the left edge $\Gamma_{L}$, we define a boundary layer function $w_{L}$. Consider the extended domain $\Omega^{* *}=(0,1) \times(-d, 1+d), 0.5>d>0$. We define $w_{L}^{*}$ to be the solution of

$$
\begin{aligned}
& L_{\varepsilon, \mu}^{* *} w_{L}^{*}=0, \quad(x, y) \in \Omega^{* *}, \\
& \left.w_{L}^{*}\right|_{\Gamma_{L}}=(u-v)^{*}, \quad w_{L}^{*}(1, y)=0, \quad y \in[-d, 1+d], \\
& w_{L}^{*}(x,-d)=w_{L}^{*}(x, 1+d)=0, \quad x \in[0,1] .
\end{aligned}
$$

Lemma 3. When $\mu^{2} \leq \frac{\gamma \varepsilon}{\alpha}$, the boundary layer function $w_{L}^{*}$ satisfies the following bounds

$$
\left|w_{L}^{*}(x, y)\right| \leq C e^{-\frac{\sqrt{\gamma \alpha}}{\sqrt{\varepsilon}} x}, \quad\left\|\frac{\partial^{i} w_{L}^{*}}{\partial y^{i}}\right\| \leq C\left(1+\sqrt{\varepsilon}^{1-i}\right), \quad i=1,2,3 .
$$

Proof. The boundary function $(u-v)(0, y)$ is extended so that $(u-v)^{*}(0, y)=$ 0 for $y<-\frac{d}{2}$ and $y>1+\frac{d}{2}$. By the comparison principle, it follows that

$$
\left|w_{L}^{*}(x, y)\right| \leq C e^{-\sqrt{\frac{\gamma \alpha}{\varepsilon}} x}, \quad(x, y) \in \bar{\Omega}^{* *} .
$$

Note that the crude derivative bounds given in Lemma 2 also apply in the case when $a_{1}(x, y) \geq 0, a_{2}(x, y) \geq 0$. Using the same argument on the extended domain we can show that these crude bounds on the derivatives also apply to $w_{L}^{*}$. In the direction orthogonal to the layer we sharpen these bounds. We first obtain a bound on $w_{L}^{*}$ to reflect the fact that it is zero on the edges $\Gamma_{T}^{* *}$ and $\Gamma_{B}^{* *}$. Note that the coefficient $a_{2}$ is extended to the domain $\Omega^{* *}$ so that $\left\|a_{2}^{*}\right\|_{\Omega^{* *}} \leq C_{1}(d+y)(1+d-y)$. Note also that

$$
L_{\varepsilon, \mu}^{* *}((d+y)(1+d-y))=-2 \varepsilon+\mu(1-2 y) a_{2}^{*}-b^{*}(d+y)(1+d-y) .
$$

Assuming that $\mu$ is sufficiently small (so that $4 C_{1} \mu<\beta$ ), it follows that

$$
\left|w_{L}^{*}(x, y)\right| \leq C(d+y)(1+d-y), \quad(x, y) \in \overline{\Omega^{* *}} .
$$

From the above bound on $\left|w_{L}^{*}(x, y)\right|$ and the fact that $w_{L}^{*}(x,-d)=w_{L}^{*}(x, 1+$ $d)=0$, we obtain

$$
\begin{aligned}
& \left|\frac{\partial w_{L}^{*}}{\partial y}(0, y)\right| \leq C, \quad \frac{\partial w_{L}^{*}}{\partial y}(1, y)=0, \\
& \left|\frac{\partial w_{L}^{*}}{\partial y}(x,-d)\right| \leq C, \quad\left|\frac{\partial w_{L}^{*}}{\partial y}(x, 1+d)\right| \leq C .
\end{aligned}
$$

Differentiate the equation $L_{\varepsilon, \mu}^{* *} w_{L}^{*}=0$ with respect to $y$ to obtain

$$
L_{\varepsilon, \mu}^{* *} \frac{\partial w_{L}^{*}}{\partial y}=-\mu \frac{\partial a_{1}^{*}}{\partial y} \frac{\partial w_{L}^{*}}{\partial x}-\mu \frac{\partial a_{2}^{*}}{\partial y} \frac{\partial w_{L}^{*}}{\partial y}+\frac{\partial b^{*}}{\partial y} w_{L}^{*}=\tilde{f} .
$$


Using the crude derivative bounds from Lemma 2 and $\mu^{2} \leq \frac{\gamma \varepsilon}{\alpha}$, we have $\|\tilde{f}\| \leq C$ and therefore

$$
\left\|\frac{\partial w_{L}^{*}}{\partial y}\right\| \leq C
$$

This argument can be extended to produce the higher derivative bounds. Using (4.1a), (4.1c) and the fact that $a_{2}^{*}(x,-d)=a_{2}^{*}(x, 1+d)=0$ we see that

$$
\begin{aligned}
& \frac{\partial^{2} w_{L}^{*}}{\partial y^{2}}(x,-d)=\frac{\partial^{2} w_{L}^{*}}{\partial y^{2}}(x, 1+d)=0, \\
& \left|\frac{\partial^{2} w_{L}^{*}}{\partial y^{2}}(0, y)\right| \leq C, \quad \frac{\partial^{2} w_{L}^{*}}{\partial y^{2}}(1, y)=0 .
\end{aligned}
$$

Using Taylor expansions and the bounds on the regular component $v$ we have the bound

$$
\left|\frac{\partial^{2} w_{L}^{*}}{\partial y^{2}}(0, y)\right| \leq C \frac{(d+y)(1+d-y)}{\sqrt{\varepsilon}} .
$$

Differentiate (4.1) twice with respect to $y$ to obtain

$$
\begin{aligned}
L_{\varepsilon, \mu}^{* *} \frac{\partial^{2} w_{L}^{*}}{\partial y^{2}}= & -2 \mu \frac{\partial a_{1}^{*}}{\partial y} \frac{\partial^{2} w_{L}^{*}}{\partial x \partial y}-2 \mu \frac{\partial a_{2}^{*}}{\partial y} \frac{\partial^{2} w_{L}^{*}}{\partial y^{2}}+\left(2 \frac{\partial b^{*}}{\partial y}-\mu \frac{\partial^{2} a_{2}^{*}}{\partial y^{2}}\right) \frac{\partial w_{L}^{*}}{\partial y} \\
& -\mu \frac{\partial^{2} a_{1}^{*}}{\partial y^{2}} \frac{\partial w_{L}^{*}}{\partial x}+\frac{\partial^{2} b^{*}}{\partial y^{2}} w_{L}^{*}=\tilde{f}_{1}, \quad(x, y) \in \Omega^{* *} .
\end{aligned}
$$

By construction the extensions $a_{1}^{*}, a_{2}^{*}$ and $b^{*}$ are such that

$$
\left|\frac{\partial^{k} a_{i}^{*}}{\partial y^{k}}\right|,\left|\frac{\partial b^{*}}{\partial y}\right| \leq C(d+y)(1+d-y), k=0,1,2 \text { and } i=1,2 .
$$

We deduce that $\left\|\tilde{f}_{1}\right\| \leq \frac{C}{\sqrt{\varepsilon}}(d+y)(1+d-y)$. Using the conditions

$$
C_{1} \mu(1+2 d)-b^{*}<0, \quad\left|a_{2}^{*}\right| \leq C_{1}(d+y)(1+d-y),
$$

we obtain

$$
\left|\frac{\partial^{2} w_{L}^{*}}{\partial y^{2}}\right| \leq \frac{C}{\sqrt{\varepsilon}}(d+y)(1+d-y) .
$$

Using this bound we obtain

$$
\begin{aligned}
& \left|\frac{\partial^{3} w_{L}^{*}}{\partial y^{3}}(x,-d)\right| \leq \frac{C}{\sqrt{\varepsilon}}, \quad\left|\frac{\partial^{3} w_{L}^{*}}{\partial y^{3}}(x, 1+d)\right| \leq \frac{C}{\sqrt{\varepsilon}} \\
& \left|\frac{\partial^{3} w_{L}^{*}}{\partial y^{3}}(0, y)\right| \leq \frac{C}{\sqrt{\varepsilon}}, \quad \frac{\partial^{3} w_{L}^{*}}{\partial y^{3}}(1, y)=0 .
\end{aligned}
$$

Differentiate (4.1) three times with respect to $y$ to obtain

$$
L_{\varepsilon, \mu}^{* *} \frac{\partial^{3} w_{L}^{*}}{\partial y^{3}}=\tilde{f}_{2}
$$


By suitable extensions $\left\|\tilde{f}_{2}\right\| \leq \frac{C}{\varepsilon}$ and then

$$
\left\|\frac{\partial^{3} w_{L}^{*}}{\partial y^{3}}\right\| \leq \frac{C}{\varepsilon}
$$

To finish, note that in the case where $4 C_{1} \mu>\beta$ and $\mu^{2} \leq \frac{\gamma \varepsilon}{\alpha}$ then $\varepsilon \geq C$ and we are in the non-singularly perturbed case in which all the derivatives are bounded independently of $\varepsilon$.

Define the boundary layer function $w_{L}$ by

$$
L_{\varepsilon, \mu} w_{L}=0,(x, y) \in \Omega,\left.\quad w_{L}\right|_{\Gamma_{L}}=u-v,\left.\quad w_{L}\right|_{\Gamma_{R}}=0,\left.\quad w_{L}\right|_{\Gamma_{T} \cup \Gamma_{B}}=w_{L}^{*} .
$$

For the boundary layer function $w_{T}$ associated with the top edge $\Gamma_{T}$, the extended domain is taken to be $(x, y) \in[-d, 1+d] \times[0,1]$ and

$$
L_{\varepsilon, \mu} w_{T}=0,(x, y) \in \Omega,\left.\quad w_{T}\right|_{\Gamma_{T}}=u-v,\left.w_{T}\right|_{\Gamma_{B}}=0,\left.w_{T}\right|_{\Gamma_{L} \cup \Gamma_{R}}=w_{T}^{*} .
$$

Lemma 4. When $\mu^{2} \leq \frac{\gamma \varepsilon}{\alpha}$, the boundary layer function $w_{T}^{*}$ satisfies the following bounds

$$
\left|w_{T}^{*}(x, y)\right| \leq C e^{-\frac{\sqrt{\gamma \alpha}}{\sqrt{\varepsilon}}(1-y)}, \quad\left\|\frac{\partial^{i} w_{T}^{*}}{\partial x^{i}}\right\| \leq C\left(1+\sqrt{\varepsilon}^{1-i}\right), \quad i=1,2,3 .
$$

Proof. The proof follows the same lines as the proof of the previous lemma. However, note that

$$
\begin{aligned}
L_{\varepsilon, \mu}^{* *} e^{-\frac{\sqrt{\gamma \alpha}}{\sqrt{\varepsilon}}(1-y)} & =\left(\gamma \alpha+\frac{\mu}{\sqrt{\varepsilon}} a_{2}^{*} \sqrt{\alpha \gamma}-b^{*}\right) e^{-\frac{\sqrt{\gamma \alpha}}{\sqrt{\varepsilon}}(1-y)} \\
& \leq\left(\gamma \alpha+\gamma a_{2}^{*}-b^{*}\right) e^{-\frac{\sqrt{\gamma \alpha}}{\sqrt{\varepsilon}}(1-y)} .
\end{aligned}
$$

Note that on the original domain $\gamma \alpha+\gamma a_{2}-b \leq 2 \gamma a_{2}-b \leq 0$. The extensions are constructed to maintain this sign pattern on the extended domain. Also, $a_{1}$ can be suitably extended so that

$L_{\varepsilon, \mu}^{* *}((x+d)(1+d-x))=\left(-2 \varepsilon+\mu(1-2 x) a_{1}^{*}-b^{*}(x+d)(1+d-x)\right) \leq 0$.

Define the boundary layer functions associated with the other two edges $w_{R}$ and $w_{B}$ analogously to $w_{T}$ and $w_{L}$. Associated with the corner $\Gamma_{L B}=$ $\Gamma_{L} \cap \Gamma_{B}$ define a corner layer function $w_{L B}$ such that

$$
\begin{aligned}
& L_{\varepsilon, \mu} w_{L B}=0, \quad(x, y) \in \Omega, \\
& w_{L B}=-w_{B}, \quad(x, y) \in \Gamma_{L}, \quad w_{L B}=-w_{L}, \quad(x, y) \in \Gamma_{B}, \\
& w_{L B}=0, \quad(x, y) \in \Gamma_{R}, \quad w_{L B}=0, \quad(x, y) \in \Gamma_{T} .
\end{aligned}
$$


Note that at the corner $(0,0), w_{L}(x, 0)$ is equal to $w_{L}(0, y)=(u-v)(0, y)$ which is equal to $(u-v)(x, 0)=w_{B}(x, 0)$ which in turn is equal to $w_{B}(0, y)$. Note also that $u-v, w_{L}, w_{B} \in C^{3, \lambda}(\bar{\Omega})$ and

$$
L_{\varepsilon, \mu} w_{L}=L_{\varepsilon, \mu} w_{B}=L_{\varepsilon, \mu}(u-v)=0 .
$$

Hence we have sufficient compatibility for $w_{L B} \in C^{3, \lambda}(\bar{\Omega})$. By using the comparison principle and the obvious barrier function, the following bound on $w_{L B}$ holds

$$
\left|w_{L B}(x, y)\right| \leq C e^{-\sqrt{\frac{\gamma \alpha}{\varepsilon}} x} e^{-\sqrt{\frac{\gamma \alpha}{\varepsilon}} y} .
$$

Associated with the corner $\Gamma_{R T}=\Gamma_{R} \cap \Gamma_{T}$ define a corner layer function $w_{R T}$ such that

$$
\begin{aligned}
& L_{\varepsilon, \mu} w_{R T}=0, \quad(x, y) \in \Omega, \\
& w_{R T}=-w_{R}, \quad(x, y) \in \Gamma_{T}, \quad w_{R T}=-w_{T}, \quad(x, y) \in \Gamma_{R}, \\
& w_{R T}=0, \quad(x, y) \in \Gamma_{L}, \quad w_{R T}=0, \quad(x, y) \in \Gamma_{B} .
\end{aligned}
$$

Noting that

$$
\begin{aligned}
L_{\varepsilon, \mu} & e^{-\frac{\sqrt{\gamma \alpha}}{2 \sqrt{\varepsilon}}(1-x)} e^{-\frac{\sqrt{\gamma \alpha}}{2 \sqrt{\varepsilon}}(1-y)} \\
& =\left(\frac{\gamma \alpha}{2}+\frac{\mu a_{1} \sqrt{\gamma \alpha}}{2 \sqrt{\varepsilon}}+\frac{\mu a_{2} \sqrt{\gamma \alpha}}{2 \sqrt{\varepsilon}}-b\right) e^{-\frac{\sqrt{\gamma \alpha}}{2 \sqrt{\varepsilon}}(1-x)} e^{-\frac{\sqrt{\gamma \alpha}}{2 \sqrt{\varepsilon}}(1-y)} \\
& \leq\left(\frac{\gamma \alpha}{2}+\frac{\gamma}{2}\left(a_{1}+a_{2}\right)-b\right) e^{-\frac{\sqrt{\gamma \alpha}}{2 \sqrt{\varepsilon}}(1-x)} e^{-\frac{\sqrt{\gamma \alpha}}{2 \sqrt{\varepsilon}}(1-y)} \\
& \leq\left(\gamma\left(a_{1}+a_{2}\right)-b\right) e^{-\frac{\sqrt{\gamma \alpha}}{2 \sqrt{\varepsilon}}(1-x)} e^{-\frac{\sqrt{\gamma \alpha}}{2 \sqrt{\varepsilon}}(1-y)} \leq 0
\end{aligned}
$$

one can establish the bound

$$
\left|w_{R T}(x, y)\right| \leq C e^{-\frac{\sqrt{\gamma \alpha}}{2 \sqrt{\varepsilon}}(1-x)} e^{-\frac{\sqrt{\gamma \alpha}}{2 \sqrt{\varepsilon}}(1-y)} .
$$

Analogous bounds hold for the other two corners. In summary we state the main result of this paper:

Theorem 1. The solution $u$ of (1.1) can be decomposed into the following sum of components

$$
u=v+w_{L}+w_{R}+w_{T}+w_{B}+w_{L B}+w_{L T}+w_{R B}+w_{R T}
$$

where $L_{\varepsilon, \mu} v=f$, and the layer and corner layer functions are each solutions of the homogeneous equation $L_{\varepsilon, \mu} w=0$. Boundary conditions for these functions can be specified so that the bounds on the components and their derivatives given below hold: 


$$
\begin{array}{ll}
\left\|\frac{\partial^{k+m} v}{\partial x^{k} \partial y^{m}}\right\| \leq C\left(1+\varepsilon^{\frac{2-k-m}{2}}\right), & 0 \leq k+m \leq 3, \\
\left|w_{L}(x, y)\right| \leq C e^{-\frac{\sqrt{\gamma \alpha}}{\sqrt{\varepsilon}} x}, & \left|w_{B}(x, y)\right| \leq C e^{-\frac{\sqrt{\gamma \alpha}}{\sqrt{\varepsilon}} y}, \\
\left|w_{R}(x, y)\right| \leq C e^{-\frac{\sqrt{\gamma \alpha}}{\sqrt{\varepsilon}}(1-x)}, & \left|w_{T}(x, y)\right| \leq C e^{-\frac{\sqrt{\gamma \alpha}}{\sqrt{\varepsilon}}(1-y)}, \\
\left|w_{L B}(x, y)\right| \leq C e^{-\frac{\sqrt{\gamma \alpha}}{\sqrt{\varepsilon}} x} e^{-\frac{\sqrt{\gamma \alpha}}{\sqrt{\varepsilon}} y}, & \left|w_{L T}(x, y)\right| \leq C e^{-\frac{\sqrt{\gamma \alpha}}{\sqrt{\varepsilon}} x} e^{-\frac{\sqrt{\gamma \alpha}}{2 \sqrt{\varepsilon}}(1-y)}, \\
\left|w_{R B}(x, y)\right| \leq C e^{-\frac{\sqrt{\gamma \alpha}}{2 \sqrt{\varepsilon}}(1-x)} e^{-\frac{\sqrt{\gamma \alpha}}{\sqrt{\varepsilon}} y}, & \left|w_{R T}(x, y)\right| \leq C e^{-\frac{\sqrt{\gamma \alpha}}{2 \sqrt{\varepsilon}}(1-x)} e^{-\frac{\sqrt{\gamma \alpha}}{2 \sqrt{\varepsilon}}(1-y)}, \\
\left\|\frac{\partial^{k} w_{L}}{\partial y^{k}}\right\| \leq C\left(1+\sqrt{\varepsilon}{ }^{1-k}\right), & \left\|\frac{\partial^{k} w_{R}}{\partial y^{k}}\right\| \leq C\left(1+\sqrt{\varepsilon}^{1-k}\right), 1 \leq k \leq 3, \\
\left\|\frac{\partial^{k} w_{B}}{\partial x^{k}}\right\| \leq C\left(1+\sqrt{\varepsilon}^{1-k}\right), & \left\|\frac{\partial^{k} w_{T}}{\partial x^{k}}\right\| \leq C\left(1+\sqrt{\varepsilon}^{1-k}\right), 1 \leq k \leq 3 .
\end{array}
$$

For all the layer components, we also have that

$$
\left\|\frac{\partial^{k+m} w}{\partial x^{k} \partial y^{m}}\right\| \leq C \varepsilon^{\frac{-k-m}{2}}, \quad 1 \leq k+m \leq 3
$$

\section{Numerical Method}

Consider the following upwind finite difference scheme

$$
L^{N, M} U\left(x_{i}, y_{j}\right)=\varepsilon \delta_{x}^{2} U+\varepsilon \delta_{y}^{2} U+\mu a_{1} D_{x}^{+} U+\mu a_{2} D_{y}^{+} U-b U=f,
$$

where $D^{+}$is the forward difference operator and $\delta^{2}$ is the standard second order centered difference operator. We apply the above finite difference operator on the tensor product mesh $\Omega^{N, M}=\Omega^{N} \times \Omega^{M}$, where $\Omega^{N}\left(\Omega^{M}\right)$ is a piecewise uniform mesh [2] that places a uniform mesh containing $\mathcal{O}(N)$ mesh points in each of the three subregions $[0, \sigma],[\sigma, 1-\sigma],[1-\sigma, 1]$. The transition points $\sigma_{x}, \sigma_{y}$ are taken to be

$$
\sigma_{x}=\min \left\{\frac{1}{4}, 2 \sqrt{\frac{\varepsilon}{\gamma \alpha}} \ln N\right\}, \quad \sigma_{y}=\min \left\{\frac{1}{4}, 2 \sqrt{\frac{\varepsilon}{\gamma \alpha}} \ln M\right\} .
$$

Remark 2. Note that if $a_{1}=a_{2}=1, \mu=0$ then $\gamma=\beta$ and the above numerical method coincides with the method analysed in [1] for the reactiondiffusion problem and shown to be parameter-uniform of second order (up to logarithmic factors).

From the pointwise bounds on the layer components and for this choice of transition point, it follows that

$$
\left\|w_{L}\left(x_{i}, y_{j}\right)\right\| \leq C N^{-2}, x_{i} \geq \sigma_{x}, \quad \text { when } \sigma_{x}<\frac{1}{4} .
$$

The discrete solution is decomposed into the sum 


$$
U=V+W_{L}+W_{R}+W_{T}+W_{B}+W_{L B}+W_{L T}+W_{R B}+W_{R T},
$$

where

$$
\begin{aligned}
& L^{N, M} V=f,\left.\quad V\right|_{\Gamma^{N, M}}=\left.v\right|_{\Gamma^{N, M}}, \\
& L^{N, M} W_{L}=0,\left.\quad W_{L}\right|_{\Gamma^{N, M}}=\left.w_{L}\right|_{\Gamma^{N, M}},
\end{aligned}
$$

and the other layer functions are defined similarly. The maximum pointwise error $\|u-U\|$ is estimated by bounding each of the error components $\| v-$ $V\|,\| w_{L}-W_{L}\|,\| w_{R}-W_{R} \| \ldots$ separately. The error $\|v-V\|$ is bounded using a classical truncation error and comparison principle argument. Using a standard truncation error argument

$$
\begin{aligned}
\left|L^{N, M}(V-v)\left(x_{i}, y_{j}\right)\right| & \leq C_{1} N^{-1}\left(\varepsilon\left\|v_{x x x}\right\|+\mu\left\|v_{x x}\right\|\right) \\
+ & C_{2} M^{-1}\left(\varepsilon\left\|v_{y y y}\right\|+\mu\left\|v_{y y}\right\|\right) \leq C\left(N^{-1}+M^{-1}\right) \sqrt{\varepsilon} .
\end{aligned}
$$

Thus at each mesh point $\left(x_{i}, y_{j}\right) \in \bar{\Omega}^{N, M}$ the regular component of the error satisfies the following parameter-uniform estimate

$$
\left|(V-v)\left(x_{i}, y_{j}\right)\right| \leq C\left(N^{-1}+M^{-1}\right) \sqrt{\varepsilon} .
$$

Lemma 5. At each mesh point $\left(x_{i}, y_{j}\right) \in \bar{\Omega}^{N, M}$, the left singular component of the error satisfies the bound

$$
\left|\left(W_{L}-w_{L}\right)\left(x_{i}, y_{j}\right)\right| \leq C\left(N^{-1} \ln N+M^{-1}\right) .
$$

Proof. Using the truncation error bounds

$$
\begin{aligned}
\left|L^{N, M}\left(W_{L}-w_{L}\right)\left(x_{i}, y_{j}\right)\right| \leq & C_{1}\left(h_{i+1}+h_{i}\right)\left(\varepsilon\left\|w_{L x x x}\right\|+\mu\left\|w_{L x x}\right\|\right) \\
& +C_{2}\left(k_{j+1}+k_{j}\right)\left(\varepsilon\left\|w_{L y y y}\right\|+\mu\left\|w_{L y y}\right\|\right)
\end{aligned}
$$

and since $\mu^{2} \leq \frac{\gamma \varepsilon}{\alpha}$, we obtain

$$
\left|L^{N, M}\left(W_{L}-w_{L}\right)\left(x_{i}, y_{j}\right)\right| \leq \frac{C_{1}}{\sqrt{\varepsilon}}\left(h_{i+1}+h_{i}\right)+C_{2} M^{-1} .
$$

The following bounds on the discrete boundary layer function $W_{L}$

$$
\left|W_{L}\left(x_{i}, y_{j}\right)\right| \leq C \prod_{s=1}^{i}\left(1+\frac{\sqrt{\gamma \alpha}}{2 \sqrt{\varepsilon}} h_{s}\right)^{-1}=\Psi_{i}
$$

are established using the discrete comparison principle and the fact that

$$
\varepsilon \delta^{2} \leq \frac{\gamma \alpha}{2} \Psi_{i+1}, \quad \mu D^{+} \Psi_{i}=-\frac{\mu \sqrt{\gamma \alpha}}{2 \sqrt{\varepsilon}} \Psi_{i+1}, \quad b \Psi_{i} \geq b \Psi_{i+1} .
$$

In the region $\left[\sigma_{x}, 1\right) \times(0,1)$ 


$$
\left|W_{L}\left(x_{i}, y_{j}\right)\right| \leq C \Psi_{N / 4} \leq C N^{-1}, \quad \sigma_{x}<1 / 4
$$

which leads to

$$
\left|\left(W_{L}-w_{L}\right)\left(x_{i}, y_{j}\right)\right| \leq C N^{-1}, \quad\left(x_{i}, y_{j}\right) \in\left[\sigma_{x}, 1\right) \times(0,1) .
$$

Note that, for $x_{i}<\sigma_{x}$, the truncation error is

$$
\begin{array}{r}
\left|L^{N, M}\left(w_{L}-W_{L}\right)\right| \leq C N^{-1} \ln N\left(\varepsilon^{3 / 2}\left\|\frac{\partial^{3} w_{L}}{\partial x^{3}}\right\|+\mu\left\|\frac{\partial^{2} w_{L}}{\partial x^{2}}\right\|\right) \\
+C M^{-1}\left(\varepsilon\left\|\frac{\partial^{3} w_{L}}{\partial y^{3}}\right\|+\mu\left\|\frac{\partial^{2} w_{L}}{\partial y^{2}}\right\|\right) .
\end{array}
$$

In the layer region $(0, \sigma) \times(0,1)$ and when $\sigma<\frac{1}{4}, h_{i}=h_{i+1}=\frac{8 \sqrt{\varepsilon}}{\sqrt{\gamma \alpha}} N^{-1} \ln N$ one can use (5.1) to obtain

$$
\left|L^{N, M}\left(W_{L}-w_{L}\right)\right| \leq C\left(N^{-1} \ln N+M^{-1}\right), \quad x_{i}<\sigma_{x} .
$$

Use an appropriately chosen barrier function and the discrete minimum principle to obtain the required result in this region. When either $\sigma_{x}=1 / 4$ or $\sigma_{y}=1 / 4$, a classical truncation error with discrete comparison principle is used to establish the error bound.

Analogous bounds hold for the error components $\left|\left(W_{B}-w_{B}\right)\right|,\left|\left(W_{R}-w_{R}\right)\right|$ and $\left|\left(W_{T}-w_{T}\right)\right|$.

Lemma 6. At each mesh point $\left(x_{i}, y_{j}\right) \in \Omega^{\bar{N}, M}$, the left, bottom and right singular components of the error satisfies the following estimates

$$
\begin{gathered}
\left|\left(W_{B}-w_{B}\right)\left(x_{i}, y_{j}\right)\right| \leq C\left(N^{-1}+M^{-1} \ln M\right) \\
\left|\left(W_{R}-w_{R}\right)\left(x_{i}, y_{j}\right)\right| \leq C\left(N^{-1} \ln N+M^{-1}\right), \\
\left|\left(W_{T}-w_{T}\right)\left(x_{i}, y_{j}\right)\right| \leq C\left(N^{-1}+M^{-1} \ln M\right) .
\end{gathered}
$$

Lemma 7. At each mesh point $\left(x_{i}, y_{j}\right) \in \bar{\Omega}^{N, M}$, the bottom-left corner singular component of the error satisfies the following estimate

$$
\left|\left(W_{L B}-w_{L B}\right)\left(x_{i}, y_{j}\right)\right| \leq C\left(N^{-1} \ln N+M^{-1} \ln M\right) .
$$

Proof. Note the truncation error bounds

$$
\begin{aligned}
\left|L^{N, M}\left(W_{L B}-w_{L B}\right)\left(x_{i}, y_{j}\right)\right| & \leq C_{1}\left(h_{i+1}+h_{i}\right)\left(\varepsilon\left\|w_{L B x x x}\right\|+\mu\left\|w_{L B x x}\right\|\right) \\
& +C_{2}\left(k_{j+1}+k_{j}\right)\left(\varepsilon\left\|w_{L B y y y}\right\|+\mu\left\|w_{L B y y}\right\|\right) \\
& \leq \frac{C_{1}}{\sqrt{\varepsilon}}\left(h_{i+1}+h_{i}\right)+\frac{C_{2}}{\sqrt{\varepsilon}}\left(k_{j+1}+k_{j}\right) .
\end{aligned}
$$

Consider the region $\Omega^{N, M} \backslash(0, \sigma) \times(0, \sigma)$. Note that 


$$
\left|W_{L B}\left(x_{\frac{N}{4}}, y_{\frac{N}{4}}\right)\right| \leq C \prod_{s=1}^{\frac{N}{4}}\left(1+\frac{4 \sigma_{x}}{N} \frac{\sqrt{\gamma \alpha}}{2 \sqrt{\varepsilon}}\right)^{-1} \prod_{r=1}^{\frac{M}{4}}\left(1+\frac{4 \sigma_{y}}{M} \frac{\sqrt{\gamma \alpha}}{2 \sqrt{\varepsilon}}\right)^{-1}
$$

In an analogous fashion to the bound on $W_{L}$, when $\sigma_{x}<1 / 4, \sigma_{y}<1 / 4$ we have

$$
\left|W_{L B}\left(x_{i}, y_{j}\right)\right| \leq C N^{-1} M^{-1}, \quad x_{1} \geq \sigma_{x} \text { and } y_{j} \geq \sigma_{y}
$$

and

$$
\left|w_{L B}\left(x_{i}, y_{j}\right)\right| \leq C N^{-2} M^{-2}, \quad x_{1} \geq \sigma_{x} \text { and } y_{j} \geq \sigma_{y} .
$$

In the region $\Omega^{N, M} \backslash\left(0, \sigma_{x}\right) \times\left(0, \sigma_{y}\right)$ one can establish

$$
\left|\left(W_{L B}-w_{L B}\right)\left(x_{i}, y_{j}\right)\right| \leq C\left(N^{-1}+M^{-1}\right), \quad \sigma_{x}<1 / 4, \sigma_{y}<1 / 4 .
$$

In the corner region $\left(0, \sigma_{x}\right) \times\left(0, \sigma_{y}\right)$, the mesh sizes are such that

$$
h_{i}=h_{i+1}=\frac{8 \sqrt{\varepsilon}}{\sqrt{\gamma \alpha}} N^{-1} \ln N, \quad k_{j}=k_{j+1}=\frac{8 \sqrt{\varepsilon}}{\sqrt{\gamma \alpha}} M^{-1} \ln M .
$$

Using the truncation error bound we obtain

$$
\left|L^{N, M}\left(W_{L B}-w_{L B}\right)\left(x_{i}, y_{j}\right)\right| \leq C\left(N^{-1} \ln N+M^{-1} \ln M\right)
$$

The proof is completed as in the case of Lemma 6 .

Similar bounds hold for the error components $\left|\left(W_{R B}-w_{R B}\right)\right|,\left|\left(W_{R T}-w_{R T}\right)\right|$ and $\left|\left(W_{L T}-w_{L T}\right)\right|$ and we conclude with the following result.

Lemma 8. Let $u$ be the solution of the differential equation (1.1) and $U$ be the discrete solution defined above. Then at each mesh point $\left(x_{i}, y_{j}\right) \in \bar{\Omega}^{N, M}$

$$
\left|(U-u)\left(x_{i}, y_{j}\right)\right| \leq C N^{-1} \ln N+C M^{-1} \ln M
$$

where $C$ is a constant independent of $\varepsilon, \mu$ and $N$.

\section{References}

[1] C. Clavero, J.L. Gracia and E. O'Riordan. A parameter robust numerical method for a two dimensional reaction-diffusion problem. Mathematics of Computation, 74, 1743-1758, 2005.

[2] P.A. Farrell, A.F. Hegarty, J.J.H. Miller, E. O'Riordan and G.I. Shishkin. Robust computational techniques for boundary layers. Chapman and Hall/CRC Press, Boca Raton, 2000.

[3] H. Han and R.B. Kellogg. Differentiability properties of solutions of the equation $-\varepsilon^{2} \triangle u+r u=f(x, y)$ in a square. SIAM J. Math. Anal., 21, 394-408, 1990.

[4] O.A. Ladyzhenskaya and N.N. Ural'tseva. Linear and Quasilinear Elliptic Equations. Academic Press, New York and London, 1968. 
[5] T. Linß and M. Stynes. Asymptotic analysis and Shishkin-type decomposition for an elliptic convection-diffusion problem. J. Math. Anal. and Applications, 261, 604-632, 2001.

[6] E. O'Riordan, M.L. Pickett and G.I. Shishkin. Parameter-uniform finite difference schemes for singularly perturbed parabolic diffusion-convection-reaction problems. Mathematics of Computation. (to appear)

[7] E. O'Riordan, M.L. Pickett and G.I. Shishkin. Singularly perturbed problems modeling reaction-convection-diffusion processes. Computational Methods in Applied Mathematics, 3(3), 424-442, 2003.

[8] H.-G. Roos and Z. Uzelac. The SDFEM for a convection diffusion problem with two small parameters. Computational Methods in Applied Mathematics, 3(3), $1-16,2003$.

[9] G.I. Shishkin. Discrete approximation of singularly perturbed elliptic and parabolic equations. Russian Academy of Sciences, Ural Section, Ekaterinburg, 1992. (In Russian)

[10] G.I. Shishkin and V.A. Titov. A difference scheme for a differential equation with two small parameters at the derivatives. Chisl. Metody Meh. Sploshn. Sredy, 7(2), 145-155, 1976. (In Russian)

[11] V.A. Titov and G.I. Shishkin. A numerical solution of a parabolic equation with small parameters multiplying the derivatives with respect to the space variables. Trudy Inst. Mat. i Meh. Ural Nauchn. Centr Akad. Nauk SSSR, 21, 38-43, 1976. (In Russian) 